\title{
Sustainable Solutions for Machine Tools
}

\author{
Eckart Uhlmann, Klaus-Dieter Lang, Lukas Prasol, Simon Thom, \\ Bernd Peukert, Stephan Benecke, Eduard Wagner, Fiona Sammler, \\ Sebastian Richarz and Nils F. Nissen
}

\begin{abstract}
Environmental, economic and social changes of any significant proportions cannot take place without a major shift in the manufacturing sector. In today's manufacturing processes, economic efficiency is realised through high volumes with the use of specialised machine tools. Change in society, such as in the form of mobility and digitisation, requires a complete overhaul in terms of thinking in the manufacturing industry. Moreover, the manufacturing industry contributes over $19 \%$ to the world's greenhouse gas emissions. As a consequence of these issues, a demand for sustainable solutions in the production industry is increasing. In particular, the concept of "cost" in manufacturing processes and thus the "system boundaries" within the production of the future has to be changed. That is, a great number of aspects to the machine tool and production technology industries can be improved upon in order to achieve a more sustainable production environment. Within this chapter, the focus lies on microsystem technology enhanced modular machine tool frames, adaptive mechatronic components, as well as on internallycooled cutting tools. An innovative machine tool concept has been developed recently, featuring a modular machine tool frame using microsystem technology for communication within the frame, which allows for a high level of flexibility. Furthermore, add-on upgrading systems for outdated machine tools-which are particularly relevant for developing and emerging countries-are poised to gain in importance in the upcoming years. The system described here enables the accuracy of outdated machine tools to be increased, thus making these machine tools comparable to modern machine tool systems. Finally, the cutting process requires solutions for dry machining, as the use of cooling lubricants is environmentally damaging and a significant cost contributor in machining processes. One such solution is the use of internally cooled cutting tools.
\end{abstract}

\footnotetext{
E. Uhlmann $(\bowtie) \cdot$ L. Prasol · S. Thom · B. Peukert · F. Sammler · S. Richarz Institute for Machine Tools and Factory Management, TU Berlin, Berlin, Germany e-mail: uhlmann@iwf.tu-berlin.de
}

K.-D. Lang · S. Benecke · E. Wagner

Research Center for Microperipheric Technologies, TU Berlin, Berlin, Germany

N.F. Nissen

Fraunhofer Institute for Reliability and Microintegration, Berlin, Germany

(C) The Author(s) 2017

R. Stark et al. (eds.), Sustainable Manufacturing, Sustainable Production,

Life Cycle Engineering and Management, DOI 10.1007/978-3-319-48514-0_4 
Keywords Flexible machine tools - Modular frames - Microsystem technology • Outdated machine tools • Adaptronic add-ons • Dry machining

\section{Introduction}

The manufacturing industry influences economic, ecological and social development worldwide. Industrial energy consumption has been increasing in most developed and undeveloped countries over the last decades. Nearly a third of the worldwide energy consumption as well as $\mathrm{CO}_{2}$-emissions are related to the production industry (International Energy Agency 2007). Furthermore, an increasingly mobile and digital society is calling for new customised technical solutions to a diverse array of products both anytime and anywhere.

In pursuit of sustainable change in the manufacturing industry, it is necessary to develop innovative solutions for machine tools as well as for production processes. To meet the demands of the global market on top of that, it's important to identify "new ways" for sustainable solutions for machine tools which may serve to generate a long-term effect in the production industry.

To impact the sustainability of machine tools, machine tool frames, in particular, must constitute a central focus. Machine tools are "static" in general. Current flexible manufacturing systems are able to handle several production situations. As a result, they are rarely fully exploited and usually "over engineered" and therein require a lot of engineering hours and raw materials. They start their product life with a negative environmental burden as a result.

Even flexible manufacturing systems are not suitable for handling the batch size "one" and therefore short development times are required that can be adapted to new requirements. These challenges are the main drivers for the future development of the machine tool industry, taking into account increasingly scarce resources at hand.

The increase in accuracy of machine tool frames, the usage of mechatronics regarding the accuracy of axes, as well as the "sustainable engineering" of solutions and recycling of components and equipment, constitute the main parts of the research work presented in this chapter. By applying modular machine tool frames using microsystem technology, the accuracy of these applications has been increased significantly. Modular machine tool frames can be used again and again and remain automatically up to date. Furthermore, the realisation of the product batch size "one" is possible if required. Through the use of adaptronic components, the accuracy of machine tools is increased, meaning older machine tools can stay abreast of latest developments. These adaptive components can furthermore be used in modular machine tool frames. By applying innovative tools for machining, sustainable solutions for machine tools are thus being identified from several different angles. 


\section{Technological Concepts}

\subsection{Microsystems Technology}

Microelectronics constitutes the core of the up-and-coming paradigm "Industrie 4.0" and "cyber physical systems," including supply chains and manufacturing environments. Small distributed systems such as wireless sensor nodes (WSN) and -systems (WSS) are mainly applied within safety systems, control systems (closed loop regulatory systems, closed loop supervisory systems, open loop control systems), monitoring systems (alerting systems, information gathering systems), e.g. the monitoring process of production equipment, yet also feature logistics support with electronic functions beyond radio-frequency identification (RFID) (Schischke 2009). The recent progress in the research of WSN application meets the requirements of manufacturing environments such as functional integrity, robustness, miniaturization and low energy consumption, as well as the more general industrial requirements of low cost, interoperability, resistance to noise and co-existence, self-configuration and organisation, scalability, data allocation and processing, resource efficient design, adaptive network optimization, time synchronization, fault tolerance and reliability, application specific design, and secure system design (Zurawski 2009). The use of WSN poses ecological questions that need to be balanced efficiently on the part of the hard- and software designers involved by means of customised WSN architecture and a WSS layout which closely follow the functional application or use-case scenario whilst at the same time maintaining a low environmental footprint.

WSN for industrial environments can be deployed remotely from the actual point of measurement using complex data acquisition techniques for gauging, constrained by environmental noise. Autonomous WSNs are applied physically in the peripheral environment of the wired grid without direct cable access. Hence, these systems enable sensing tasks at frequently changing or remote locations that cannot be accessed by conventional measurement equipment (Ovsthus and Kristensen 2014). Basic WSN hardware design features a central processing unit (microcontroller) that is linked to memory, a communication unit (radio frequency (RF) transceiver including an antenna), sensor units, an independent energy source (energy harvester or battery) and, optionally, a human-machine-interface (HMI).

In the case of distributed WSNs, the energy efficiency is a critical factor, as they carry limited energy storage. While wired concepts allow the focus to be on the quality of service (QoS), wireless concepts aim to achieve primarily power conservation at the expense of, for example, lower throughput or higher transmission delay. To meet the requirements of energy efficiency while conserving reliable messaging, industrial settings call for an application of specific communication technologies including RF interference problems, more complex circuitry, individual software algorithm design, WSN topology, such as star or multihop meshed communication network and costs. An overview of radio frequencies involved is given in (Rault et al. 2014). Data losses and communication reliability that appear 
in industrial settings suffer from noise, co-channel interferences and multipath propagation resulting from such typical obstacles as stationary or moving objects (noise) and RF interferences from other devices (Ovsthus and Kristensen 2014).

Wireless sensor networking technology in terms of protocols and standards for the so-called industrial internet of things (IIoT) attempt to combat the obstacles mentioned (Hu 2015). Based on the IEEE 802.XX standard, the derivations ISA 100 and wireless HART are used for applications in process automation since they are considered to be energy efficient, robust and reliable. ZigBee (IEEE 15.4) is considered to be geared towards low energy consumption, low cost and security. As a middleware publishing/subscribing protocol, the Message Queuing Telemetry Transport (MQTT) protocol is being considered for reliable messaging due to its lightweight architecture (Sheltami et al. 2015).

With greatly reduced energy consumption, it is also becoming feasible to employ efficient battery and energy harvesting technology as decentralised energy sources. Primary batteries offer the highest performance with about $3000 \mathrm{~J} / \mathrm{cm}^{3}$. Manufacturing environments offer high potential for broadband vibration, fluidic or thermoelectric energy harvesting sources at about $40 \mu \mathrm{W} / \mathrm{cm}^{2}$ to $1 \mathrm{~mW} / \mathrm{cm}^{2}$. In the trade-off, high performance computing and high sampling rates stand up against low-power and miniaturised applications (Beeby 2006, Gungor and Hancke 2009; Elvin and Erturk 2013).

A broader investigation of the trade-off between environmental benefits and the negative impact of the additional microsystems including wireless sensor nodes (WSNs) was conducted in the German technology assessment study "Innovationsund Technikanalyse Autonomer Verteilter Mikrosysteme" (Autonomous Distributed Sensor Systems) (Schischke 2009). Qualitative results show that primary effects, e.g. resource consumption or recycling, are mainly negative, while indirect impact, such as production efficiency, are positive. Moreover, the long-term compatibility between the different lifecycles and concepts of machine tool components and electronics remain an interesting research topic.

To date, a link between the impact of microsystems on component level and modularity leading to further improvement recommendations on the system level remains missing. The question of how to support the designer of electronic systems with easy-to-use indicators while addressing sustainability issues has been addressed by (Wagner et al. 2016). The design methodology developed serves to connect common electronic components to their contained materials and selected impact types like cumulative energy demand or recyclability. From the system assessment standpoint, there is a need for evaluations of the trade-off between more functionality and more resource impact for $25+$ years use time.

\subsection{Reconfigurable Machine Tools}

The design process of machine tools represents a major investment in tangible and intangible resources for machine tool manufacturer and consumer. Lead times are 
especially high in the case of individualised machine tools and assembly lines. From a sustainability perspective, long lifecycles of machines of over 25 years are difficult to manage, given the volatility of product variants and low batch sizes of today's global market. As the production of machine tools has high relevance in the world economy (Verein Deutscher Werkzeugmaschinenfabriken e.V 2015), machine tool manufacturers are therefore interested in shortening delivery times, increasing flexibility and reducing material consumption in pursuit of ultimately offering superior solutions to customers.

Moving on to machine tool frames, these elements provide fundamental structural support for every machine tool. Their production requires expensive engineering, testing and high precision manufacturing. As conventionally casted or welded structures, machine tool frames are limited in terms of reconfiguration and cannot be altered after manufacturing. This restricts the reuse to configurations which were initially incorporated into the planning on the part of the engineers.

As the public is becoming increasingly aware of the issue of sustainability, sustainable product manufacturing has become a selling point of its own right. This is true for products manufactured with machine tools, but also for machine tools as products themselves. Previous manufacturing paradigms aimed at producing homogenous products at highest qualities and lowest costs. Nowadays, consumers are demanding the production of individualised goods. This manufacturing paradigm is called mass customisation, which among other developments, most recently led to research on reconfigurable machine tools (RMT) within reconfigurable manufacturing systems (RMS).

Reconfigurable Machine Tools Reconfigurable Manufacturing was defined by Koren et al. (1999), and pointed towards the need for scalable and adaptable manufacturing equipment. One solution to enable the necessary shifts in manufacturing paradigms is the introduction of RMT, made from different modules. With this concept, the foundations of the modular design of machine tools were studied extensively and compiled by Ito (2008). Pioneer work in the modularity of machines was done by Herrmann and Brankamp (1969), who defined the idea of Building Block Systems (BBS). Since then, many research and industrial activities regarding modularisation and reconfiguration in manufacturing (tool design) have been carried out, some of which have found their way into industrial application.

Mori and Fujishima (2009) have presented designs of reconfigurable CNC machine tools addressing the design concept, machine tool configuration and application examples, respectively. The design of the machine tools allowed for selecting a number of axes by the individual axis modules and reconfiguration of the spindle in horizontal and vertical directions. Wulfsberg et al. (2013) give a summary of the concepts developed in the context of modularity in small machine tools for micro-production. The design measures are associated with those of conventional machine tool components for the development of modular systems. Scalability of the working area, namely the change in size of the working area, was achieved within the research work presented. Abele and Wörn (2009) describe a catalogue of components developed for reconfigurable machine tools in the project METEOR. A new approach was developed with the "Reconfigurable Multi 
technology Machine tool" (RMM) concept that enables the integration of multiple production functions in a single workspace. Most related to sustainability issues, the German project LOeWe (German acronym for Life cycle Oriented development of machine tools) aimed at designing a modular machine tool capable of serving as the basis for different manufacturing processes by including aspects like use-phases and the corresponding life-cycles (Denkena et al. 2006).

With a higher degree of modularisation, challenges have arisen particularly for modularised machine tool frames due to the decreased stiffness which results. The mechanical module interfaces represent serial compliances, reducing the overall rigidity of the given structure assembled. Of course, rigidity is one of the key factors for high productive manufacturing. At the same time, however, the stability of a machine tool mainly depends on a sufficient level of dynamic stiffness of the frame. A common approach for improving the dynamic behaviour is found in the inclusion of actuators and control loops within machine tool structures for the purpose of enhancing damping or for decreasing the dynamic compliance. A building block system for modular machine tool frames therefore requires individualised sensors and actuators.

In addition to sensor technology (see section Microsystems technology), actuators are of great interest when designing for sustainability. As the paradigm of sustainable product design has emerged over the last decades, the design of actuator systems needs to take multifaceted aspects of sustainability into consideration accordingly. This includes avoidance or at least reduction of energy consumption, the substitution of hazardous materials with environment-friendly ones, and low-cost solutions for the production of actuators, leading to solutions for Green Engineering and Manufacturing (Dornfeld 2012).

Various research work on this topic exists, e.g. analysing the energy efficiency of hydraulic, pneumatic and piezoelectric actuators and improving on those actuators (Eriksson 2007; Harris et al. 2014). Existing approaches for achieving higher efficiency vary depending on the actuating principles involved. The most common approach for improving sustainability has turned out to be downsizing, featuring a combination and reconstruction of systems. A combination of different actuation principles, on the other hand, improves the energy efficiency of actuators and combines the advantages of both principles. In this context, for example, hybrid drives are being designed which provide lower energy consumption than regular linear motor direct drives and combine their higher speed and accuracy with the higher damping of screw drives (Okwudire and Rodgers 2013). Chen et al. (2014) present the design of a novel three-degrees-of-freedom linear magnetic actuator which increases the damping and static stiffness of flexible structures during machining. The actuator uses electromagnetic materials which allow larger load capacity and almost no hysteresis compared to piezo and magnetostrictive materials such as Law et al. (2015) report on a novel electro-hydraulic actuator that attenuates and isolates ground motion to keel dynamic excitations transmitted to machine tools below permissible levels. The analysis of optimal placement of actuators can also lead to increased efficiency and thus has to be taken into consideration (Okwudire and Lee 2013). 
Applying this principle into the context of RMT, sustainability benefits are anticipated by designing building block systems using tiered technological architectures. Passive lightweight modules can be used to provide structural integrity. Meanwhile, adaptable and reusable sensor technology can increase the smartness of the building block system and improve the overall machine tool frame performance in combination with actuating modules and closed loop controls algorithms.

\subsection{Adaptronics in Machine Tools}

One of the major limiting factors for the machining quality at high cutting speeds is the static and dynamic behaviour of a machine tool (Ast et al. 2007). The challenge of achieving high static and dynamic stiffness and implementing lightweight design requires adaptronic solutions, which allow for the direct influence of the structural properties of mechanical structures. Adaptronic systems can be integrated into machine tools for different purposes, e.g. active error compensation, active vibration control and active chatter avoidance. The integration of adaptronic systems into machine tools stands as a (key) enabler for achieving higher machining performance as well as for reducing resource consumption, emissions and costs in manufacturing.

A key example of such adaptronic systems is found in dual-stage feed drives. Dual-stage feed drives designed for the purpose of allowing high precision positioning over a large workspace on conventional machine tools, fast tool servos (FTS) (e. g. piezoelectric actuator driven flexures) are connected in a series with a machine tool drive in a so-called dual-stage feed drive (DSFD) setup. Woronko et al. (2003) implemented a piezo-based FTS for precision turning on conventional CNC lathes. The results show that the tool positioning accuracy as well as the surface quality could be increased. Elfizy et al. (2005) investigated DSFD for milling processes in pursuit of enabling high precision positioning over a large workspace. In that process, a two-axis flexure mechanism featuring piezoelectric actuators is connected in a series with the machine tool drive stage. The tracking error for sinusoidal profile milling was reduced by approximately $80 \%$ compared to a single stage feed drive. In addition, Drossel et al. (2014) show versatile applications of adaptronic systems in machining processes. FTS systems are currently applied in the form of honing processes for the purpose of increasing the positioning accuracy and for reducing vibration of the tool. The achievable shape accuracy could be therein improved to $\pm 3 \mu \mathrm{m}$ and the surface roughness decreased to a reduced peak height of $\mathrm{R}_{\mathrm{pk}}=1.7 \mu \mathrm{m}$ (Drossel et al. 2014).

\section{Active vibration control}

Although active vibration control is not a new concept, a recent development in the field of chatter avoidance for machine tools by means of active damping was discussed by Brecher et. al. (2013). Hömberg et al. (2013) investigated the influences on chatter and solutions for chatter avoidance to improve the efficiency of 
production of high quality parts at higher removal rate. Ast et al. (2007) integrated an adaptronic rod in a lightweight structure of a lambda kinematics machine tool in order to overcome vibrations at the tool centre point (TCP), which were identified as a limiting performance factor. Moreover, the active component is designed in a modular manner in such a way that it is transferable to comparable machine tools.

Structure integrated adaptronic components were introduced by Brecher and Manoharan (2009). These devices can compensate deformations of slider structures. Quasi-static and dynamic compensation can be designed for translational and/or rotational axes (Abele et al. 2008; Aggogeri et al. 2013). The modularity is addressed by designing a single unit, which can be used as an active workpiece holder or as a device mounted on a spindle for vibration control (Aggogeri et al. 2013). Chen et al. (2014) presented a smart way of orienting electromagnetic actuators by obtaining two translational and one rotational degree of freedom for an active workpiece holder. Real time compensation of geometric deviations is provided by employing rigid body simulations implemented in the form of an observer in CNC-control (Denkena et al. 2014).

Control strategies With developments in control theory, sophisticated and 'easy to implement' control strategies have evolved over time. To that end, Tiwari et al. (2015) presented an investigation of the application of artificial intelligence techniques, such as fuzzy logic, neurofuzzy, genetic algorithm, genetic programming and data mining in the mechanical engineering domain. An Artificial Neural Network (ANN)-based system of identification and control of dynamic systems was proposed in the late 1980s and early 1990s (Narendra and Parthasarathy 1990). Thereafter, many applications based on neural network control were developed. For instance, a control based on two neural networks with a radial basis function was proposed by Liu and Fuji (2014) for precise positioning of a system with piezoelectric actuators.

\section{Upgrade on demand}

The requirements on machine tools depend on and change with the manufacturing task at hand, which is a challenge in a low batch size production environment, see Fig. 1. Machine tools which do not meet the required properties are considered outdated and need a technical overhaul in order to produce productively.

In the machine tool sector, retrofitting is a common principle in the pursuit of reviving outdated machine tools. Retrofitting is primarily understood as steady modification of an existing machine tool and comprises activities such as turning-off or replacing components of machine tools to save energy, or the exchanging out of key wear parts (Gontarz et al. 2012). These activities target a machine tool, its auxiliary systems or the machining process itself.

Among the concept add-ons presented here are optional equipment for upgrading specific functions of machine tools in a flexible manner. The conceptual distinction between retrofit and upgrade by means of add-ons is that the application of add-ons is not permanent but flexible to the required specifications. Sharing add-ons for a pool of machine tools thus enables a production environment to be more resource efficient. 


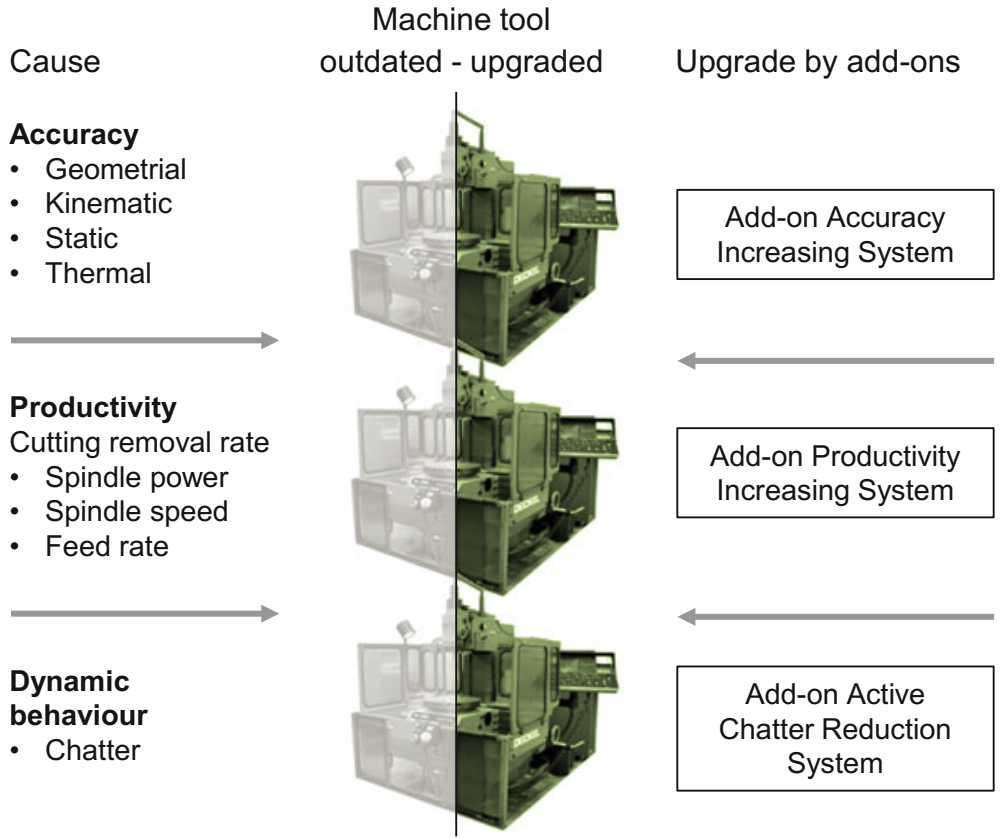

Fig. 1 Concept of machine tool upgrade by add-ons

Easy-to-install capabilities are a necessary feature of add-ons in order to apply the systems in a flexible manner. In addition to the mechanical fixing (e.g. by releasable fastener) on a machine tool, the add-ons applied should be independent of the machine tool control thus allowing the upgrading of a wide range of machines.

\subsection{Dry Machining}

Whenever cooling liquid (CL) is used in the production process, tools and workpieces are cooled directly and the friction between the two of them is reduced. This leads to improved tool life and a better workpiece surface quality at the same time. Another advantage is that all chips are washed out of the working area (Klocke and Eisenblatter 1997). If CL is employed in the production process, the following disadvantages have to however be accepted - usually, the chips are contaminated with CL and must be cleaned in downstream installations such as centrifuges and briquetting machines. Moreover, occupational-safety measures have to be applied in order to limit CL's harmful effects to workers' health (Klocke and Eisenblatter 1997; Byrne et al. 2003; Heisel and Lutz 1993a, b). 
Dry processing is a production technique characterised by low-power consumption and lean manufacturing chains, as there is no need for the production, monitoring and disposal of cooling lubricants (Ward et al. 2016). This both saves on downstream cleaning and eliminates potential working time lost due to sickness caused by contact with cooling lubricants. However, no cooling of the cutting edge takes place, and the positive effects of lubrication in an interrupted cutting process are likewise lost. In a worst-case scenario, a switchover to dry processing will involve downward revision of the wet processing cutting parameters, which means that the savings on cooling lubricants and the benefits of lean manufacturing processes are offset by a loss in productivity. Alternatively, cooling can be affected with compressed air, solid carbon dioxide or liquid nitrogen (Uhlmann et al. 2012). These alternative cooling agents volatilise in the machining zone, yet their application comes with such high costs that only in exceptional cases do they stand as economically reasonable options (Uhlmann et al. 2012, 2016). Another low-cost tool cooling solution is found in closed internal cooling systems which use a heat sink for dispersing the tool's machining heat in a cooling medium. The accumulated heat in the tool is directed away, and thus is separated from the rise in temperature during actual cutting time.

\section{Sustainable Solutions}

This chapter describes the development of three sustainable production technology concepts as shown in Fig. 2, schematically. The concepts and solutions for sustainable manufacturing technology presented in Sect. 2 require a step-by-step change within this discipline. In this process, the first step to be realised lies in the integration of innovative machining processes into existing machine tools. In the second step, the modularization of machine tool frames is to be realised, taking into account smart microsystem technology and innovative machining processes. In the final step, the upgrading of the machine tools can be undertaken as necessary.

Internally-cooled tools Turning tools with closed internal cooling systems must meet a different set of requirements compared to conventional tools. In particular, they must ensure mechanical stability under the temperature range of the deployed cooling medium. An internally-cooled turning tool and cooling periphery was, furthermore, developed within the framework of the work described here.

LEG $^{2}$ O smart building block system As Koren et al. (1999) stated, RMT are anticipated to contribute significantly to the manufacturing of mass-customised products. Key attributes related to the sustainability of RMT result from delivering the machining purpose when and as needed and from avoiding downtime or underutilization. Although modularization was achieved on the hardware side of machine tools, among various other components in the past, the modularisation of the machine tool frame remains an open question of great relevance. A Smart Building Block System (BBS) for modular machine tool frames is envisioned to overcome technological limitations and to provide a sustainable alternative to the 


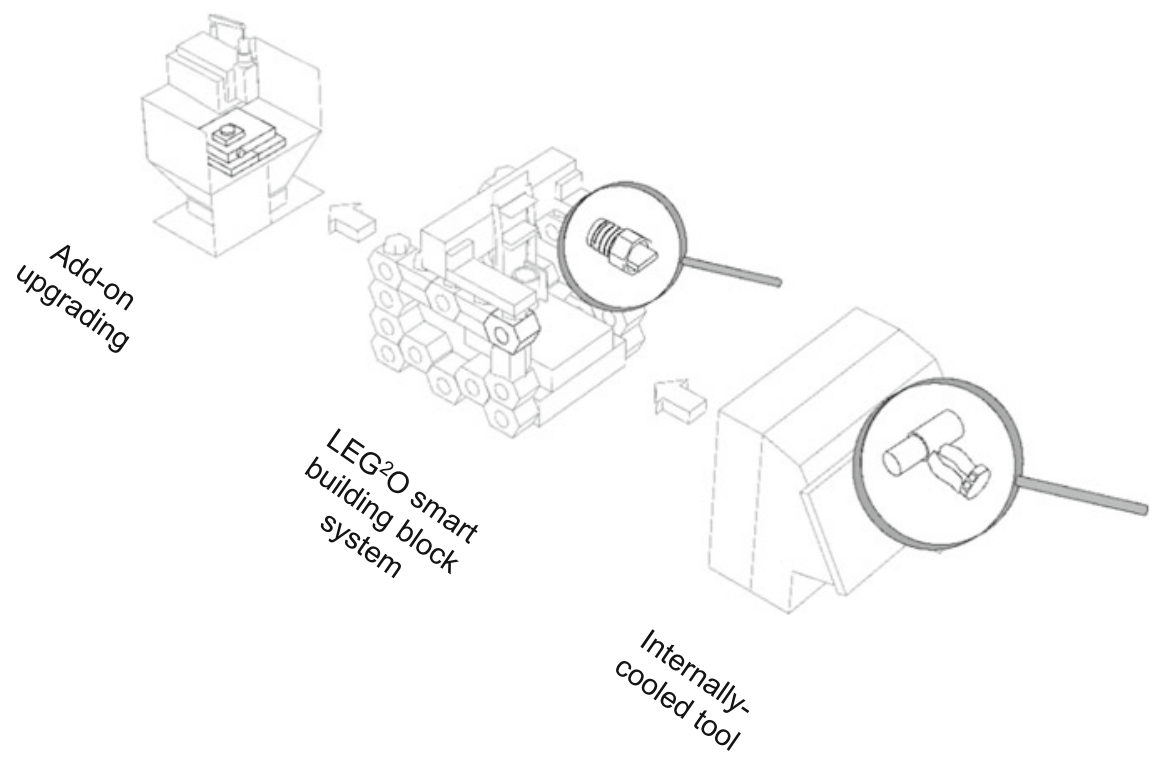

Fig. 2 Sustainable machine tool concepts-development from the present to the future

design of conventional machine tools. This is achieved by using a tiered technological approach combining microsystem technology (MST) and mechatronic technology (MT).

Add-ons for outdated machine tools Compared to current machine tools, outdated machine tools suffer from high positioning deviations and fail to add sufficient value to current production systems (Uhlmann and Kianinejad 2013). Under this concept, this problem is tackled by an upgrade using an Add-on Accuracy Increasing System (AAIS). The add-on systems are used without exchanging essential system components. By upgrading or enhancing the functionality of outdated machine tools rather than replacing them with new machines, valuable resources can be saved (Allwood et al. 2011). The add-on solution developed, furthermore helps to keep outdated machine tools competitive in the contemporary production chain by increasing not only positioning accuracy but also by allowing for active vibration control to reduce chatter (Kianinejad et al. 2016).

\subsection{Smart Building Block Systems}

The technological basis of the $\mathrm{LEG}^{2} \mathrm{O}$ BBS (German acronym for lightweight and accuracy optimised) consists of passive, active and MST enhanced smart building blocks, called modules, with a weight limitation of $30 \mathrm{~kg}$ per unit. In accordance with worker safety regulations for manual lifting, each component of the $\mathrm{LEG}^{2} \mathrm{O}$ 
BBS allows for manual handling during assembly, maintenance and upgrading with low-level infrastructure (Steinberg and Windberg 2011). Studies on use-cases of these BBS led to the conclusion that for $\mathrm{LEG}^{2} \mathrm{O}$ BBS, most cutting machine tool scenarios are sound (Peukert et al. 2013).

What's more, first order impacts directly associated with $\mathrm{LEG}^{2} \mathrm{O}$ BBS were addressed in a tiered life cycle sustainability assessment (Peukert et al. 2015b). The research revealed clear benefits during the whole life cycle compared to conventional static machine tool frames due to reuse and adaptation of machine tools.

Passive modules provide fundamental mechanical properties, e.g. rigidity, to support the core structure of modular machine tool frames. Within the scope of sustainable design, resource efficiency was set as a target during the design phase of the passive module. A bionic-inspired fractal design approach was chosen, resulting in high geometric flexibility with stiff and scalable structures based on two modules with a hexagonal prism shape (Peukert et al. 2015a). These modules were topologically optimised to provide a lightweight and resource-efficient foundation with the necessary rigidity to compete with conventional machine tool frames. Figure 3 shows the development history of the different design stages of passive modules and shows the topologically optimised hexagons with a weight of 6.2 and $12.3 \mathrm{~kg}$, respectively. Yet, on top of advantages in damping behaviour, decreased rigidity and dynamic stiffness are caused by the high number of joints (Uhlmann and Peukert 2015), leading to the need for active modules and compensation.

Smart modules The physical instantiation of MST by means of wireless sensor nodes, provides smart functionalities (e.g. orientation sensing) in dedicated passive modules within the frame, or so-called smart modules. Data on module identification as well as parameters related to the physical state of the machine tool frame are shared between smart modules and one centralized receiving unit using wireless communication. The demand for fully autonomous concepts and long-term usage called for a revised catalogue of requirements apart from conventional industrial sensors. As the overall system size of highly miniaturised sensors is dominated by the autonomous energy supply, measures for reducing the average power consumption were identified as crucial aspects of system design (Lambebo 2014). With highly efficient programming routines, e.g. reduced instruction sets and the relocation of dedicated tasks into the base stations, operating times were even further prolonged without the need for battery replacement. However, only through the application of advanced packaging technologies can optimised form factors be
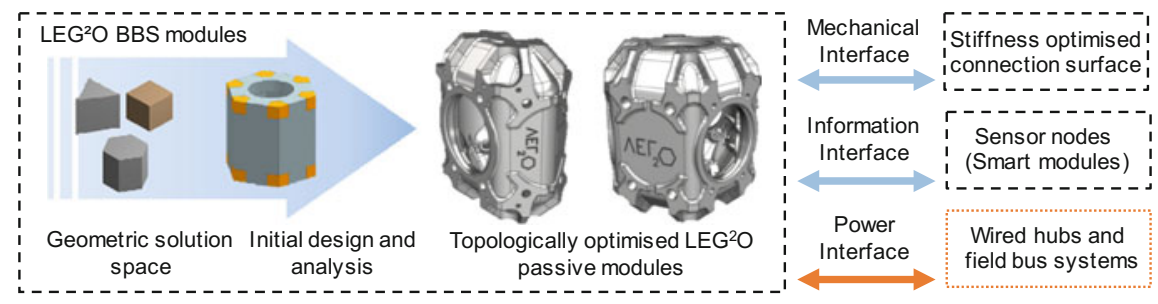

Fig. 3 Topologically optimised passive modules and interfaces 
achieved that allow for the least interference at maximum functional density and long lifetime at the workstation. A combined approach including Flip Chip assembly, surface mount technology and a sequence of embedding processes on panel level was furthermore used to realise a new generation of WSNs. Physical devices demonstrating the fusion of MST hardware with MT (e.g. in a screw) are shown in Fig. 4 with selected functional groups indicated.

Active modules are used to compensate for displacements. The control loop of the actuators uses on-line data, transmitted wirelessly by smart modules. Apart from the machine tool dynamic, thermal loads constitute a main cause of inaccuracies with machine tool frames. Hence, the active modules have a control platform manipulated by three separate compliant mechanisms. The compliant mechanisms are driven by the thermal deformation of aluminium bars controlled by thermoelectric modules. The slow nature of thermal deviations allows the usage of solid state relays to power numerous active modules from a single power supply. Hence, additional sustainability benefits can be achieved in comparison to traditional piezo-driven approaches, which require one amplifier per channel. At a simplified level, the construction and usability of this approach, as well as the concomitant sustainability benefits and control of the active module, were analysed (Uhlmann and Peukert 2015). The topology of the mechanism facilitates a self-adapting passive compensation movement at the output platform by change of ambient temperature due to an inherent thermal compensation of approximately $\mathrm{x}_{\mathrm{i}} \approx 2 \mu \mathrm{m} / \mathrm{K}$. The actuator is designed to provide a compensating range of $\Delta \mathrm{x}=100 \mu \mathrm{m}$ maintaining micrometer accuracy. Figure 5 shows the actuator and experimental results of the closed loop control in a prototypical test structure.

A combined hard- and software infrastructure synchronises all relevant data for the analysis of thermal distortion of the frame as shown in Fig. 6. Additional data sets, e.g. module identification and orientation, are distributed to external devices for visualisation on a tablet PC. A methodology was developed to support the spatial setup of WSNs within the $\mathrm{LEG}^{2} \mathrm{O}$ BBS (Uhlmann et al. 2014). This method supports the optimisation of the relationship between the mechanical structure,
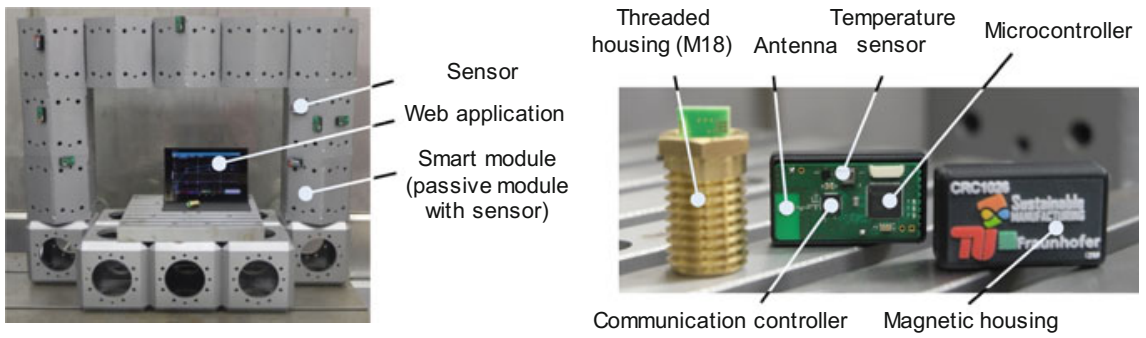

Fig. 4 Wireless sensor nodes for parameter monitoring of passive $\mathrm{LEG}^{2} \mathrm{O}$ modules - the final technology demonstrator of the sensor fits into a hollow M18 screw 


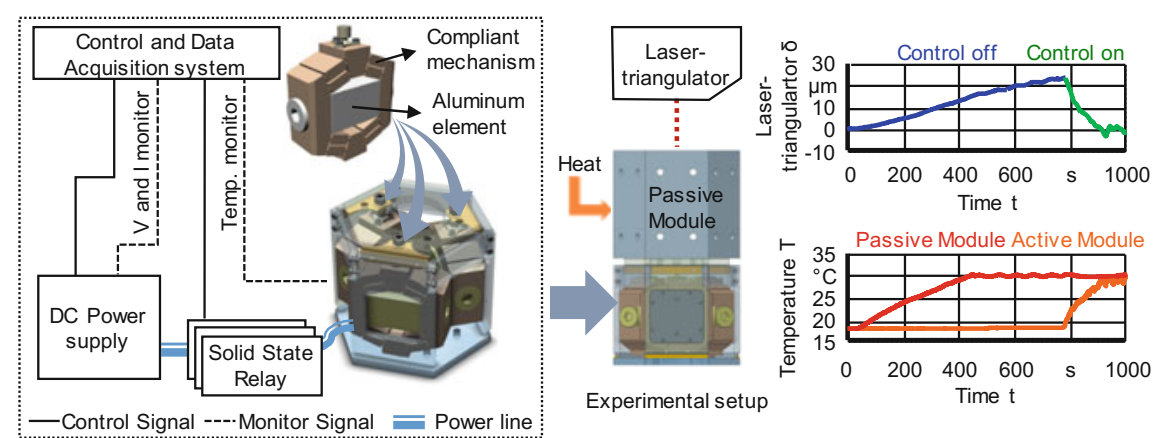

Fig. 5 Active module for compensation of thermal deviations

loading scenario and the number of sensor nodes, and thus helps minimize the utilization of MST.

\subsection{Add-Ons for Machine Tool Upgrade}

Outdated machine tools suffer from high positioning deviations compared to current machine tools and fail to add sufficient value to present production systems (Uhlmann and Kianinejad 2013). Within the scope of the work described here, this technical obsolescence is addressed by an upgrade using an Add-on Accuracy Increasing System (AAIS). The add-on systems are used without exchanging essential system components. By upgrading or enhancing the functionality of outdated machine tools rather than replacing them with new machines, valuable resources can be saved (Allwood et al. 2011). The add-on solution developed helps to keep outdated machine tools competitive in the contemporary production chain by increasing not only positioning accuracy, but also by allowing for active vibration control to reduce chatter (Kianinejad et al. 2016).

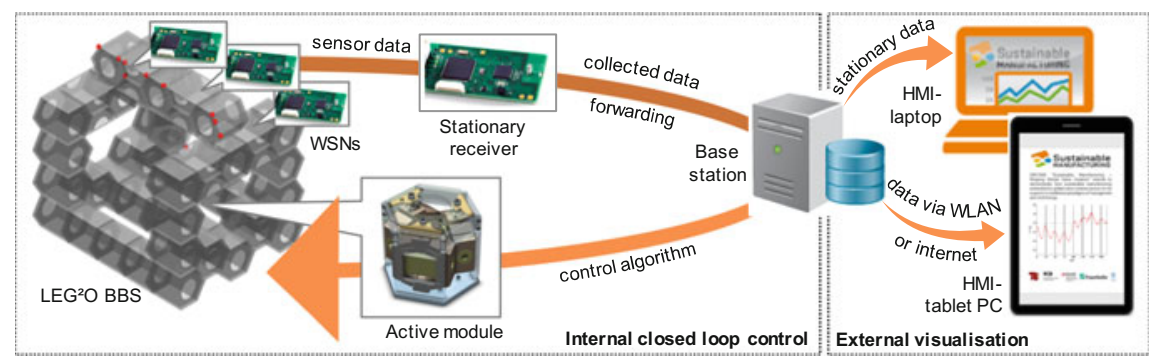

Fig. 6 Concept of the smart modules equipped with MST (WSNs) and IT infrastructure (Base station, HMI) 
The energy efficiency for different machining operations of an outdated milling machine and a modern one was compared in (Kianinejad et al. 2015). Though the energy consumption of the outdated machine exceeds that of the newer one, the upgrade of an outdated machine tool presented saves raw materials, energy for material extraction, along with manufacturing energy by not replacing the outdated milling machine with a new one.

Add-on Accuracy Increasing System (AAIS) A high accuracy error compensation table has been integrated and tested on a representative milling machine tool, shown as Fig. 7. The FP4NC milling machine, Friedrich Deckel AG, München, Germany is run by a GRUNDIG Dialog 4 control, so that compensation by control unit is not possible. Sensors of the add-on system can measure a significant portion of static, dynamic, and kinematic inaccuracies. These measurements are used, together with a feedback control mechanism to correct the errors by means of piezoelectric actuators. The add-on error compensation table is run by separate control hardware (dSPACE 1103) and does not share the control unit of the machine tool, making the solution developed both modular and independent of the type of machine. In order to use the error compensation table depending on the respective manufacturing tolerances and to allow sharing with different machine tools, the main challenge with the integration of an error compensation table is to provide easy-to-install capabilities. These capabilities are achieved by independent control, as only one interface is required to the machine tool for sensor readouts, along with a screw connection of the table, see also (Kianinejad et al. 2016).

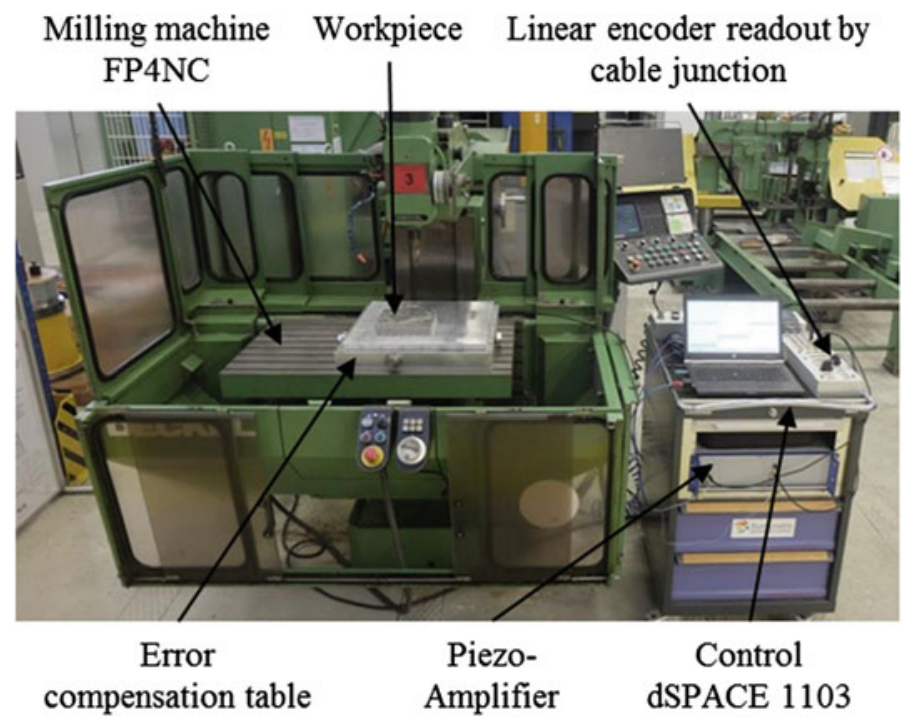

Fig. 7 Test setup of a upgraded milling machine FP4NC, Friedrich Deckel AG, München, Germany 
Fig. 8 Error compensation table (clamping plate removed)

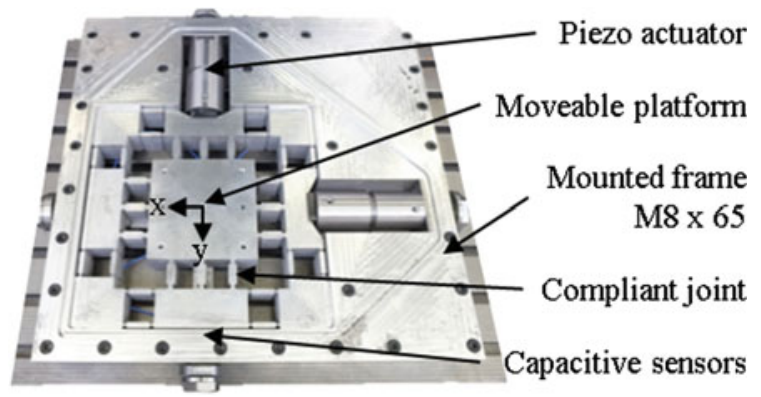

The error compensation table corrects the relative position between workpiece and tool in real time and provides compensation in two perpendicular axes in the horizontal plane (see Fig. 8). A capacitive sensor mounted in the frame is used in each direction to detect the motion of the platform with respect to the frame. Along with measuring the quasi-static position, these sensors also measure the dynamic movement of the platform. Together with the piezo actuators and control designed, active damping is also provided by AAIS.

The piezo actuators are pre-stressed by a housing in order to protect them against forces. The nominal stroke of the piezo actuators $\mathrm{s}_{\mathrm{A}}=125 \mu \mathrm{m}$ is reduced by the applied pre-stress and voltage of amplifiers, so that AAIS can provide a compensation of $\mathrm{s}_{\text {AAIS }}=55 \mu \mathrm{m}$ in each of the axes.

To overcome the problem of stick-slip and backlash, the error compensation table is designed to be monolithic, featuring compliant joints which provide high stiffness up to $\mathrm{k}_{\mathrm{z}}=100 \mathrm{~N} / \mu \mathrm{m}$ in the vertical direction (Kianinejad et al. 2016). In order to provide high strain by low stress in the compliant joints, aluminum alloy (AW7075) was chosen due to its high ratio of strength to elastic modulus.

A look-up table is generated containing the repeatable error by initial measurements on the linear positioning of the machine in the $\mathrm{x}$ and $\mathrm{y}$ axes. By feeding the look-up table with the reference position ( $x, y)$ of the linear encoders of FP4NC, Friedrich Deckel AG, München, Germany a reference signal is generated. This reference signal is then fed to the control of the xy-table for tracking.

Figure 9 shows the static positioning test performed by a laser interferometer on the $\mathrm{x}$ axis of FP4NC with and without the AAIS and also compares the data to the positioning accuracy of a modern machine tool, a DMU50, DMG MORI AG, Bielefeld, Deutschland produced in 2008. It can be seen that AAIS improves the positioning accuracy significantly.

\subsection{Internally-Cooled Tools}

The tool design of turning tools was reconsidered when taking the requirements and conditions of an integrated closed-loop cooling system for turning tools into 
Fig. 9 Positioning deviations of the $\mathrm{x}$-axis

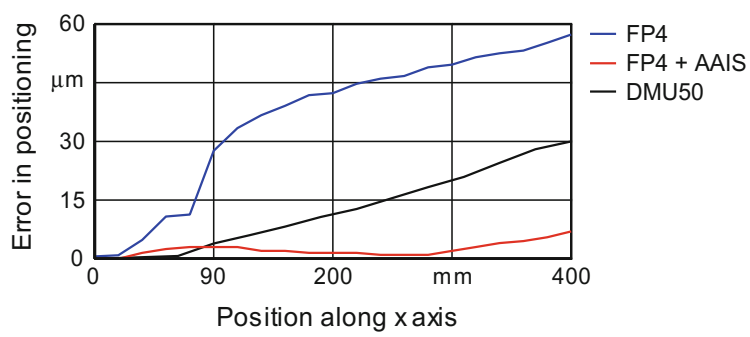

consideration, e.g. cooling medium temperatures from -210 to $+40{ }^{\circ} \mathrm{C}$. As a result, topology optimised tool geometries were simulated and investigated. The resulting tool design concepts were assessed by taking into account the weight, stiffness, and the level of integration (Uhlmann et al. 2014). The position of the cutter should likewise avoid shifting during the operation of the cooling system, as this otherwise results in marked variations in the geometry of the finished components. To manage this, the cooling medium channels are integrated into the turning tool holder body and are decoupled from its support structure. Manufacturing of flow-optimised cooling channels is difficult, thus selective laser melting is used for fabrication of the tool holder, see Fig. 10.

Comparison of different cooling strategies A comprehensive analysis of three different cooling methods compared to a dry machining process was carried out with a variation of different cutting speeds $\mathrm{v}_{\mathrm{c}}$ :

- Dry machining with an internally-cooled tool with a water-ethanol mixture as process coolant

- Dry machining with an internally-cooled tool with liquid nitrogen as process coolant

- Flood cooling

The energy demand of these cooling methods compared to a dry machining process is given in Fig. 11. The use of liquid nitrogen as a process coolant improves the tool lifetime by at least $50 \%$. However, due to the temperature influence of the coolant, the TCP shows a displacement of up to $\mathrm{dT}=0.2 \mathrm{~mm}$. The total energy
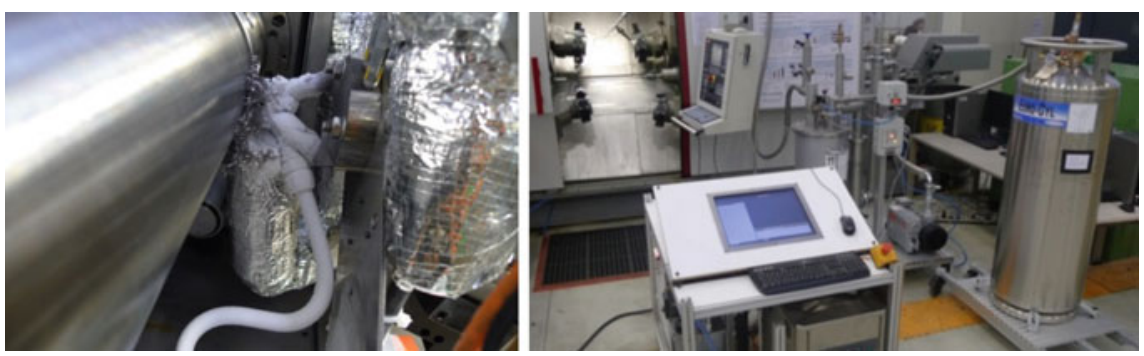

Fig. 10 Internally-cooled turning tool and cooling periphery 


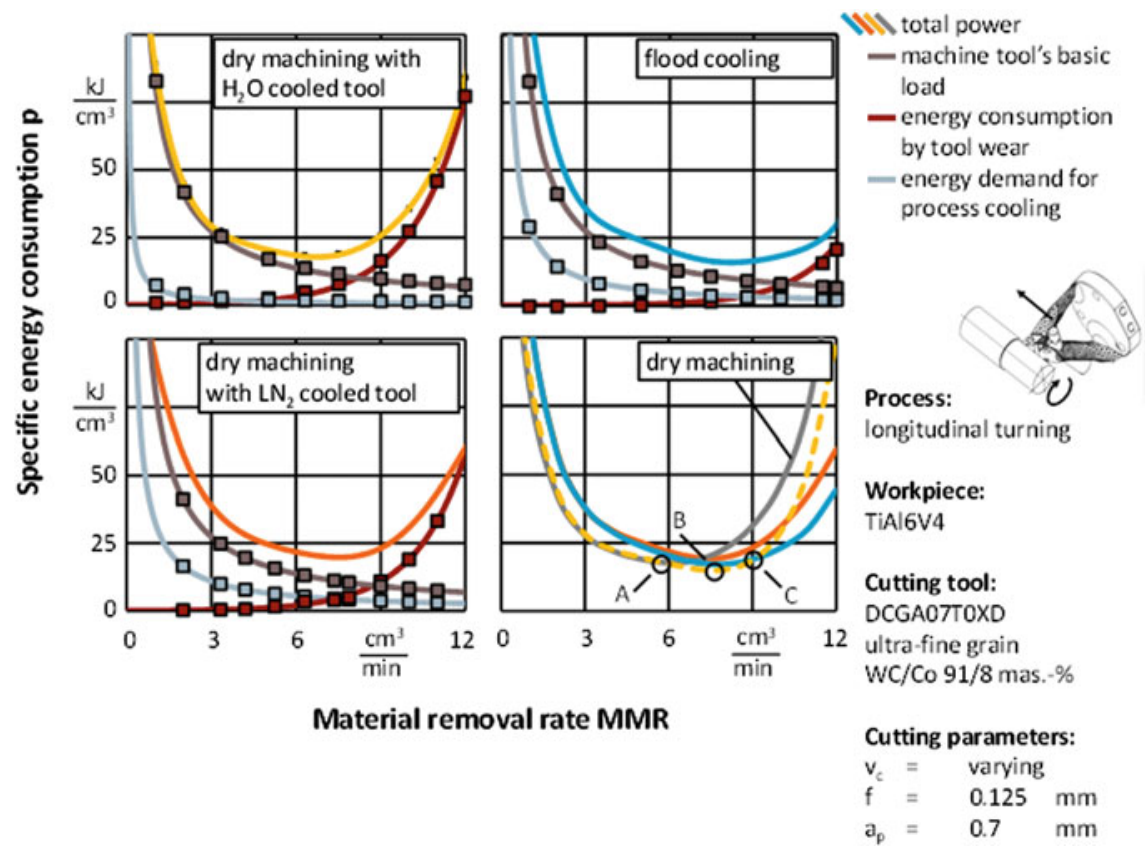

Fig. 11 Energy demand of cutting processes with different cooling methods

demand is comparable to flood cooling. Using the water-ethanol mixture, the tool lifetime can be improved by at least $40 \%$ while the TCP shows no thermal displacement. In addition, the process yields the highest energy efficiency (see point B in Fig. 11).

In summary, the need for process and tool cooling depends on the chosen process parameters. The highest energy efficiency for part finishing can be achieved with dry machining (point A), for semi-finishing with indirect cooled tools (point $\mathrm{B}$ ), and for very high material removal rates with flood cooling (point C).

\section{Conclusion}

To conclude, this chapter shows the ongoing research in pursuit of a sustainable impact on the worldwide production industry by means of the development of innovative solutions for manufacturing environments. A special focus is placed on concepts for reconfigurable machine tools: where fluctuating production environments cannot be tackled by conventional static constructions, a modular building block system is designed that enables the production of homogenous products at high quality standards, lowest costs and featuring the option for partial replacement, repair, exchange, or upgrade with regular service intervals, therein avoiding 
downtime or underutilization. The BBS concept encompassed various perspectives for overcoming technological limitations. The technological basis of the proposed $\mathrm{LEG}^{2} \mathrm{O}$ BBS consists of a scalable structure, where connections, interfaces and microsystem technology constitute elements for connecting and enabling the application of two basic module geometries of a hexagonal prism shape, involving active modules that allow for the compensation of thermal deformations. In this process, the actuator of different actuation principles as well as optimal placement within the machine tool frame serve together to improve energy efficiency and combines the advantages of the principles. In addition, passive modules provide stiffness and serve as the structural base.

Smart modules are enhanced by microsystem technology, namely wireless sensor nodes. Battery and energy harvesting technology in combination with highly efficient programming routines and customised hardware architecture allow for autonomous and flexible hard- and software infrastructure to synchronise all relevant data for the analysis of thermal distortion. Adaptive components then increase the accuracy of machine tools while remaining up-to-date.

The increase of the accuracy of machine tool frames, the usage of mechatronics regarding the accuracy of axes, as well as the "sustainable engineering" of solutions and recycling of components and equipment constitute the main parts of the research work at hand. Detail questions for the future concern communication reliability, data losses, environmental trade-offs between benefits and impact, as well as evaluation approaches for 25+ years of use time. A link between the microsystems' impact on component level and modularity is so far still missing. Moreover, long-term compatibility between the different lifecycles and concepts of machine tool components and electronics needs to be investigated. Though modularisation was achieved on the hardware side of machine tools among various components, the net effect on sustainability of the modularisation of the machine tool frame remains an open question.

By upgrading outdated machine tools with add-on components, the accuracy of modern machine tools is achieved. Sensors of the add-on system can measure a significant portion of static, dynamic, and kinematic inaccuracies. These measurements are applied together with a feedback control to correct the errors through the use of piezoelectric actuators.

By upgrading or enhancing the functionality of older machine tools, valuable resources and energy consumption can be saved. This aim is realised without exchanging essential system components, but by using high precision compensation add-on systems. Sensors of the add-on system are able to measure a portion of static, dynamic and kinematic inaccuracies. The add-on compensation is realised by a separate control unit, making the development solution modularised and easily incorporated into different types of machine tools. 


\section{References}

Abele, E., and A. Wörn. 2009. Reconfigurable machine tools and equipment. In Changeable and reconfigurable manufacturing systems, 111-125. Springer.

Abele, E., H. Hanselka, F. Haase, D. Schlote, and A. Schiffler. 2008. Development and design of an active work piece holder driven by piezo actuators. Production Engineering 2(4): 437-442. doi:10.1007/s11740-008-0123-3.

Aggogeri, F., F. Al-Bender, B. Brunner, M. Elsaid, M. Mazzola, A. Merlo, D. Ricciardi, M. de la O Rodriguez, and E. Salvi. 2013. Design of piezo-based AVC system for machine tool applications. Mechanical Systems and Signal Processing 36(1): 53-65. doi:10.1016/j.ymssp. 2011.06.012.

Allwood, J.M., M.F. Ashby, T.G. Gutowski, and E. Worrell. 2011. Material efficiency: A white paper. Resources, Conservation and Recycling 55(3): 362-381. doi:10.1016/j.resconrec.2010. 11.002 .

Ast, A., S. Braun, P. Eberhard, and U. Heisel. 2007. Adaptronic vibration damping for machine tools. CIRP Annals-Manufacturing Technology 56(1): 379-382. doi:10.1016/j.cirp.2007.05. 088.

Beeby, S. 2006 Energy harvesting vibration sources for wireless sensor networks with special focus on vibrations.

Brankamp, K., and J. Herrmann. 1969. Baukastensystematik-Grundlagen und Anwendung in Technik und Organisation. Ind.-Anz 91: 693-697.

Brecher, C., and D. Manoharan. 2009. Aktive Dämpfung für Portalmaschinen: Entwicklung adaptronischer Kompensationsmodule für Schieberstrukturen. wtWerkstattstechnik online 99 (5): 288-293.

Brecher, C., S. Bäumler, and B. Brockmann. 2013. Avoiding chatter by means of active damping system for machine tools. Journal of Machine Engineering 13(3): 117-128.

Byrne, G., D. Dornfeld, and B. Denkena. 2003. Advanced cutting technology. Annals of the CIRP-Manufacturing Technology 52(2): 483-507.

Chen, F., X. Lu, and Y. Altintas. 2014. A novel magnetic actuator design for active damping of machining tools. International Journal of Machine Tools and Manufacture 85: 58-69. doi:10. 1016/j.ijmachtools.2014.05.004.

Denkena, B., et al. 2006. Life-cycle oriented development of machine tools. In 13th cooperative institutional research program international conference on life cycle engineering, 693-698.

Denkena, B., L. Overmeyer, K.M. Litwinski, and R. Peters. 2014. Compensation of geometrical deviations via model based-observers. The International Journal of Advanced Manufacturing Technology 73(5-8): 989-998. doi:10.1007/s00170-014-5885-5.

Dornfeld, D.A. 2012. Green manufacturing: fundamentals and applications. Springer Science \& Business Media.

Drossel, W.-G., A. Bucht, C. Hochmuth, A. Schubert, A. Stoll, J. Schneider, and R. Schneider. 2014. High performance of machining processes by applying adaptronic systems. Procedia CIRP 14: 500-505.

Elfizy, A.T., G.M. Bone, and M.A. Elbestawi. 2005. Design and control of a dual-stage feed drive. International Journal of Machine Tools and Manufacture 45(2): 153-165. doi:10.1016/j. ijmachtools.2004.07.008.

Elvin, Niell, Erturk, Alper (Eds.). 2013. Advances in energy harvesting methods. doi:10.1007/9781-4614-5705-3.

Eriksson, B. 2007. Control strategy for energy efficient fluid power actuators. Lic Thesis, LiU-Tryck, Linköping. 
Gontarz, A., F. Hänni, L. Weiss, and K. Wegener. 2012. Machine tool optimization strategies: Evaluation of actual machine tool usage and modes. In Sustainable manufacturing, ed. Günther Seliger, 131-36. Berlin: Springer.

Gungor, V.C., and G.P. Hancke. 2009. Industrial wireless sensor networks: Challenges, design principles, and technical approaches. IEEE Transactions on Industrial Electronics 56(10): $4258-4265$.

Harris, P., S. Nolan, and G.E. O'Donnell. 2014. Energy optimisation of pneumatic actuator systems in manufacturing. Journal of Cleaner Production 72: 35-45.

Heisel, U., and M. Lutz. 1993a. Probleme der umwelt- und humanverträglichen Fertigung am Beispiel der Kühlschmierstoffe - Erster Teil, DIMA, 93/8.9:81-83.

Heisel, U., and M. Lutz. 1993b. Probleme der umwelt- und humanverträglichen Fertigung am Beispiel der Kühlschmierstoffe - Zweiter Teil. DIMA 93(10): 35-40.

Hömberg, D., E. Uhlmann, O. Rott, and P. Rasper. 2013. Development of a stability prediction tool for the identification of stable milling processes. In Process machine interactions, ed. B. Denkena, and F. Hollmann, 203-224. Springer.

$\mathrm{Hu}, \mathrm{P} .2015$. A system architecture for software-defined industrial Internet of Things. In IEEE international conference on ubiquitous wireless broadband (ICUWB). Paris: IEA Publications.

International Energy Agency. 2007. Tracking industrial energy efficiency and $\mathrm{CO}_{2}$ emissions. Paris: IEA Publications.

Ito, Y. 2008. Modular design for machine tools. McGraw Hill Professional.

Kianinejad, K., E. Uhlmann, and B. Peukert. 2015. Investigation into energy efficiency of outdated cutting machine tools and identification of improvement potentials to promote sustainability. Procedia CIRP 26: 533-538.

Kianinejad, K., S. Thom, S. Kushwaha, and E. Uhlmann. 2016. Add-on error compensation unit as sustainable solution for outdated milling machines. Procedia CIRP 40: 174-178.

Klocke, F., and G. Eisenblatter. 1997. Dry cutting. Annals of the CIRP-Manufacturing Technology 46(2): 519-526.

Koren, Y., et al. 1999. Reconfigurable manufacturing systems. CIRP Annals-Manufacturing Technology 48(2): 527-540.

Lambebo, A., and S. Haghani. 2014. A wireless sensor network for environmental monitoring of greenhouse Gases. In ASEE 2014 zone I conference.

Law, M., et al. 2015. Active vibration isolation of machine tools using an electro-hydraulic actuator. CIRP Journal of Manufacturing Science and Technology 10: 36-48.

Liu, D., and F. Fuji. 2014. An adaptive internal model control system of a piezo-ceramic actuator with two RBF neural networks. In 2014 IEEE international conference on mechatronics and automation (ICMA), 210-215. doi:10.1109/ICMA.2014.6885697.

Mori, M., and M. Fujishima. 2009. Reconfigurable machine tools for a flexible manufacturing system. In Changeable and reconfigurable manufacturing systems, 101-109. Springer.

Narendra, K.S., and K. Parthasarathy. 1990. Identification and control of dynamical systems using neural networks. IEEE Transactions on Neural Networks/A Publication of the IEEE Neural Networks Council 1(1): 4-27. doi:10.1109/72.80202.

Okwudire, C., and J. Lee. 2013. Minimization of the residual vibrations of ultra-precision manufacturing machines via optimal placement of vibration isolators. Precision Engineering 37(2): 425-432.

Okwudire, C., and J. Rodgers. 2013. Design and control of a novel hybrid feed drive for high performance and energy efficient machining. CIRP Annals-Manufacturing Technology 62(1): 391-394.

Ovsthus, K., and Lars M. Kristensen. 2014. An industrial perspective on wireless sensor networks - a survey of requirements, protocols, and challenges. IEEE Communications Surveys \& Tutorials 16(3): 1391-1412. 
Peukert, B., J. Mewis, M. Saoji, E. Uhlmann, S. Benecke, R. Thomasius, N. F. Nissen, and K.-D. Lang. 2013. Microsystem enhanced machine tool structures to support sustainable production in value creation networks.

Peukert, B., M. Saoji, and E. Uhlmann. 2015a. An evaluation of building sets designed for modular machine tool structures to support sustainable manufacturing. Procedia CIRP 26: 612-617.

Peukert, B., S. Benecke, J. Clavell, S. Neugebauer, N. F. Nissen, E. Uhlmann, K.-D. Lang, and M. Finkbeiner. 2015b. Addressing sustainability and flexibility in manufacturing via smart modular machine tool frames to support sustainable value creation. Procedia CIRP 29: 514 519.

Rault, T., A. Bouabdallah, and Y. Challal. 2014. Energy efficiency in wireless sensor networks: A top-down survey. Computer Networks 67: 104-122.

Schischke, K. 2009. Innovations- und Technikanalyse autonomer verteilter Mikrosysteme: Schlussbericht. Berlin, Hannover: Technische Informationsbibliothek u. Universitätsbibliothek.

Sheltami, T., A. Al-Roubaiey, A. Mahmoud and E. Shakshuki. 2015. A publish/subscribe middleware cost in wireless sensor networks: A review and case study. In IEEE 28th Canadian conference on electrical and computer engineering (CCECE).

Steinberg, U., and H.-J. Windberg. 2011. Heben und Tragen ohne Schaden. Dortmund: Baua.

Tiwari, A., R. Jaideva, and S. K. Pradhan. 2015. An investigation into use of different soft artificial intelligence techniques in mechanical engineering domain. In Proceedings of fourth international conference on soft computing for problem solving, ed. K.N. Das, K. Deep, M. Pant, J.C. Bansal and A. Nagar, 521-541. Springer.

Uhlmann, E., and K. Kianinejad. 2013. Investigation of the upgrading potentials of out-of-date cutting machine tools to promote sustainable and global value creation. In Proceeding of the 11th global conference on sustainable manufacturing, ed. G. Seliger, 574-579. Berlin: University Publisher of TU Berlin.

Uhlmann, E., and B. Peukert. 2015. Erhöhen der Dämpfung hohler Leichtbaustrukturen: Ein passiver und nachhaltiger Ansatz zur Verbesserung der Strukturdämpfung. Wt Werkstattstechnik online 105(7/8): 446-450.

Uhlmann, E., P. Fürstmann, M. Roeder, S. Richarz, and F. Sammler. 2012. Tool wear behaviour of internally cooled tools at different cooling liquid temperatures. In Proceedings of the 10th global conference on sustainable manufacturing, ed. G. Seliger, and E. Kılıç, 21-27. Berlin: University Publisher of TU Berlin.

Uhlmann, E., M. Saoji, S. Kushwaha, and B. Peukert. 2014. Optimierte Sensorplatzierung in Mess-Netzwerken. Werkstattstechnik online 5: 266-271.

Uhlmann, E., M. Saoji, and B. Peukert. 2016. Utilization of thermal energy to compensate quasi-static deformations in modular machine tool frames. Procedia CIRP 40: 1-6.

Verein Deutscher Werkzeugmaschinenfabriken e.V. 2015. Marktbericht 2014: Die deutsche Werkzeugmaschinenindustrie und ihre Stellung im Weltmarkt [Online]. http://www.vdw.de/. Accessed 11 April 2016.

Wagner, E., S. Benecke, J. Winzer, N.F. Nissen, and K.-D. Lang. 2016. Evaluation of indicators supporting the sustainable design of electronic systems. Procedia CIRP 40: 469-474.

Ward, H., M. Burger, Y. Chang, P. Fürstmann, S. Neugebauer, A. Radebach, G. Sproesser, A. Pittner, M. Rethmeier, E. Uhlmann, J. Steckel. 2016. Assessing carbon dioxide emission reduction potentials of improved manufacturing processes using multiregional input output frameworks. Journal of Cleaner Production. doi:10.1016/j.jclepro.2016.02.062. 
Woronko, A., J. Huang, and Y. Altintas. 2003. Piezoelectric tool actuator for precision machining on conventional CNC turning centers. Precision Engineering 27(4): 335-345. doi:10.1016/ S0141-6359(03)00040-0.

Wulfsberg, J. P., A. Verl, K.-H. Wurst, S. Grimske, C. Batke, and T. Heinze. 2013. Modularity in small machine tools. Production Engineering 7(5): 483-490.

Zurawski, R. 2009. Wireless sensor network in industrial automation. In International conference on embedded software and systems, Pittsburgh, 2009.

Open Access This chapter is licensed under the terms of the Creative Commons Attribution 4.0 International License (http://creativecommons.org/licenses/by/4.0/), which permits use, sharing, adaptation, distribution and reproduction in any medium or format, as long as you give appropriate credit to the original author(s) and the source, provide a link to the Creative Commons license and indicate if changes were made.

The images or other third party material in this chapter are included in the book's Creative Commons license, unless indicated otherwise in a credit line to the material. If material is not included in the book's Creative Commons license and your intended use is not permitted by statutory regulation or exceeds the permitted use, you will need to obtain permission directly from the copyright holder.

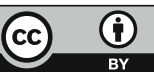

